Journal of Molecular Structure, 32 (1976) 379-392

oElsevier Scientific Publishing Company, Amsterdam - Printed in The Netherlands

\title{
STRUCTURES OF THE STRAINED MOLECULES HEXAMETHYLETHANE AND 1,1,2,2-TETRAMETHYLETHANE BY GAS-PHASE ELECTRON DIFFRACTION
}

\author{
L. S. BARTELL* and T. L. BOATES** \\ Department of Chemistry, Iowa State University, Ames, Iowa 50010 (U.S.A.) and \\ Department of Chemistry, University of Michigan*, Ann Arbor, Michigan 48104 (U.S.A.)
}

(Received 11 July 1975)

\begin{abstract}
Hexamethylethane has bond lengths of $r_{\mathrm{g}}(\mathrm{C}-\mathrm{C}$ central $)=1.582 \pm 0.01 \AA, r_{\mathrm{g}}(\mathrm{C}-\mathrm{C}$ terminal $)=1.542 \pm 0.002 \mathrm{~A}, r_{\mathrm{g}}(\mathrm{C}-\mathrm{H})=1.113 \pm 0.004 \mathrm{~A}$, and bond angles of $\angle \mathrm{C}_{\mathrm{c}} \mathrm{C}_{\mathrm{c}} \mathrm{C}_{\mathrm{t}}=$ $111.0 \pm 0.3^{\circ}$ and $\angle \mathrm{CCH}=111.5 \pm 1.4^{\circ}$ (uncertainties $2 \sigma$ ). It suffers a mean twist from $D_{\text {id }}$ symmetry of $5 \pm 4^{\circ}$. Tetramethylethane is approximately $60 \%$ gauche, $40 \%$ trans, in composition in the gas-phase with bond lengths $r_{g}(C-C$ central, trans $)=1.544 \pm 0.006 \AA$, $r_{\mathrm{g}}(\mathrm{C}-\mathrm{C}$ central, gauche $)-r_{\mathrm{g}}(\mathrm{C}-\mathrm{C}$ central, trans $)=0.002 \mathrm{~A}$ (assumed), $r_{\mathrm{g}}(\mathrm{C}-\mathrm{C}$ terminal) $=1.539 \pm 0.002 \AA, r_{\mathrm{E}}(\mathrm{C}-\mathrm{H}$ ave $)=1.115 \pm 0.004 \AA$, and angles are distributed around the average angle of $111.3_{5} \pm 0.4^{\circ}$ in accord with a picture of steric interactions. The gauche conformer is twisted $65 \pm 5^{\circ}$ from the eclipsed configuration. Amplitudes of vibration were determined for both molecules. The structure of $\left(\mathrm{CH}_{3}\right)_{3} \mathrm{BN}\left(\mathrm{CH}_{3}\right)_{3}$ is considered in the light of $\left(\mathrm{CH}_{3}\right)_{3} \mathrm{CC}\left(\mathrm{CH}_{3}\right)_{3}$ results and it is concluded that the $\mathrm{B}-\mathrm{N}$ length is intermediate between the values proposed by Lide and by Geller.
\end{abstract}

\section{INTRODUCTION}

As part of a continuing study of steric interactions in hydrocarbons, an investigation of the molecular structures of 1,1,2,2-tetramethylethane and hexamethylethane was undertaken. Although preliminary results have been referred to in several publications $[1,2]$, certain features of the structures warrant discussion. In particular, the molecules exhibit steric deformations and illustrate the limited utility of conventional rules of thumb about additivity of gauche methyl-methyl interactions. Hexamethylethane also provides information helping to resolve the structure of its isoelectronic analog, $\left(\mathrm{CH}_{3}\right)_{3} \mathrm{NB}\left(\mathrm{CH}_{3}\right)_{3}$, about which contradictory interpretations of the microwave spectrum have been advanced $[3,4]$.

In the case of hexamethylethane, two separate and completely independent determinations were made on sets of data acquired over a year apart. This affords an unusual opportunity to assess the precision and reproducibility

\footnotetext{
* Author and address to which correspondence should be mailed. **Present address, Bemidji State College, Bemidji, Minnesota 56601, U.S.A.
} 
of the electron diffraction method as applied to a molecule offering significant difficulties.

\section{EXPERIMENTAL PROCEDURE}

A sample of hexamethylethane was obtained from the American Petroleum Institute. Its proton NMR spectrum revealed no impurities at a level approaching $1 \%$. Tetramethylethane with a specified purity greater than 99.8\% was purchased from the Phillips Petroleum Company. Patterns of both compounds were obtained with the diffraction unit [5] constructed at Iowa State University and later transferred to the University of Michigan. Experimental procedures for recording and measuring patterns have been described elsewhere [5].

The structure determination of hexamethylethane was repeated partly because the first plates taken with the $21-\mathrm{cm}$ camera were considerably darker than we consider optimum $(0.45<A<1.4)$ and, perhaps in part because of this, the background at small $s$ had a much more exaggerated hump in it than is normally encountered. The intensities of both hexamethylethane and tetramethylethane represent measurements from four plates from each camera distance for each run*.

\section{ANALYSIS OF DATA}

Structure parameters were derived primarily by least-squares analyses of radial distribution curves, though intensity curves for hexamethylethane were also analyzed. Since comparatively delicate analyses of correlated parameters were involved, the $f_{\mathrm{N}}(r)$ variant rather than the $f_{\mathrm{C}}(r)$ variant of the radial distribution method was adopted, as described elsewhere $[5,6]$. Preliminary comparisons in the case of hexamethylethane confirmed the superior stability of the $f_{\mathrm{N}}(r)$ approach. The final radial distribution curves, which are plotted in Figs. 1 and 2, were based on a Degard damping function $\exp \left(-0.00073 s^{2}\right)$.

\section{Hexamethylethane}

If the carbon skeleton is assumed to have $D_{3}$ symmetry and the methyl groups, $C_{3 \mathrm{v}}$ symmetry, the structure of hexamethylethane may be described with seven parameters. The seven parameters chosen for refinement were:

(1) $\bar{r}$, the average $\mathrm{C}-\mathrm{C}$ bond length,

(2) $\Delta r$, the difference between the central and terminal bond lengths,

\footnotetext{
* The experimental leveled intensities for hexamethylethane and tetramethylethane at camera distances 21,11 and $7 \mathrm{~cm}$. are available as SUB. PUB. NO. 26028 (13 pages) from BLL. These data show that the defect found on run 1 of hexamethylethane is absent in run 2.
} 




Fig. 1. Radial distribution curve for hexamethylethane, run II. Solid curve, experimental; dotted curve, calculated.

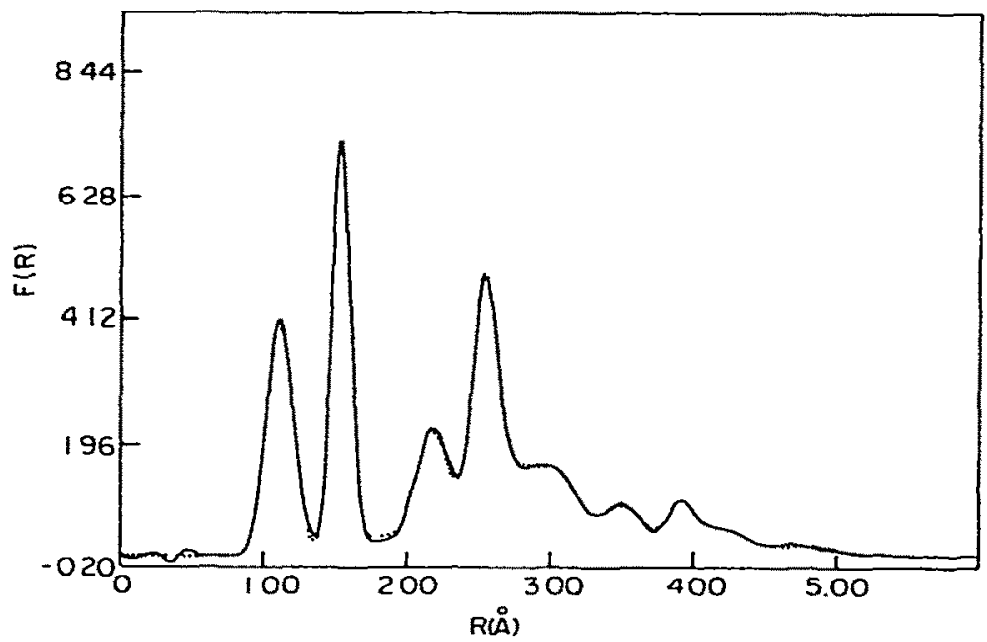

Fig. 2. Radial distribution curve for tetramethylethane. Solid curve, experimental; dotted curve, calculated.

(3) $r_{\mathrm{CH}}$, the $\mathrm{C}-\mathrm{H}$ bond length,

(4) $\alpha_{\mathrm{CCC}}$, the angle between central and terminal $\mathrm{C}-\mathrm{C}$ bonds,

(5) $\alpha_{\mathrm{CCH}}$, the $\mathrm{CCH}$ bond angle,

(6) $\tau_{C}$, the torsional displacement of one tertiary group with respect to the other, taken as zero in the $D_{3 d}$ staggered form, and

(7) $\tau_{M}$, the torsional displacement of methyl groups about terminal $\mathrm{C}-\mathrm{C}$ bond, taken as zero when the $\mathrm{C}-\mathrm{H}$ bonds are staggered with respect to the $\mathrm{C}-\mathrm{C}$ bonds. 
The two torsional parameters $\tau_{\mathbf{C}}$ and $\tau_{M}$ are determined only marginally by the data and, therefore, they were not varied simultaneously with the other parameters in the least-squares analyses. An estimation of these parameters was made by following the standard deviation between the experimental and theoretical $f_{\mathrm{N}}(r)$ curves at various imposed parameter values. The two parameters were fixed at certain values while the other five parameters were allowed to vary in a least-squares analysis. Results are plotted in Fig. 3. For $\tau_{M}$ there is no observable deviation from perfect staggering. There seems to be, however, a significant distortion of the molecule from $D_{3 \mathrm{~d}}$ symmetry. In subsequent least-squares investigations on the intensity, $\tau_{C}$ and $\tau_{M}$ were fixed at the values $5^{\circ}$ and $0^{\circ}$, respectively, found as described above.

Since there were only six well-resolved peaks in the radial distribution curve, only six amplitudes of vibration were varied in the least-squares analysis, in accord with the following limitations:

(1) The amplitude of vibration of the central bond was assumed to be $0.001 \AA$ greater than the terminal bond amplitudes (difference estimated by an extension [7] of Badger's rule [8]).

(2) When two nonbonded $\mathrm{C} \cdot \mathrm{C}$ distances differed by less than their amplitude of vibration, they were constrained to have the same amplitude.

(3) The amplitudes of all $\mathrm{H} \cdots \mathrm{H}$ nonbonded distances and all $\mathrm{C} \cdot \mathrm{H}$ nonbonded distances greater than $2.3 \AA$ were fixed at plausible values ranging from $0.13 \AA$ to $0.20 \AA$.

The results of the two runs and the two analyzing techniques are given in Tables 1 and 2. Parameter correlation coefficients are listed in Table 3 . In the least-squares analyses of the intensity curves, the individual camera ranges were treated separately. Because this approach seriously limits the assessment of amplitudes of vibration, the amplitudes were not varied in the intensity analyses but were fixed at the values determined in the analysis of $f_{N}(r)$ curves. Bastiansen-Morino shrinkage corrections [9] applied in the data analysis were estimated crudely; these values are indicated in Tables 1 and 2.
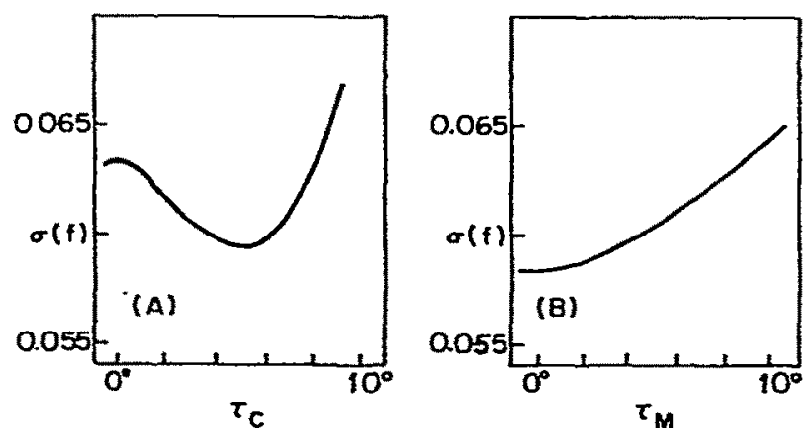

Fig. 3. Closeness of fit of radial distribution function $f_{N}(r)$ as a function of torsion parameters $\tau_{C}$ and $\tau_{M}$. Curve $(A), \tau_{M}$ fixed at $5^{\circ}$. Curve $(B) r_{C}$ fixed at $5^{\circ}$. 


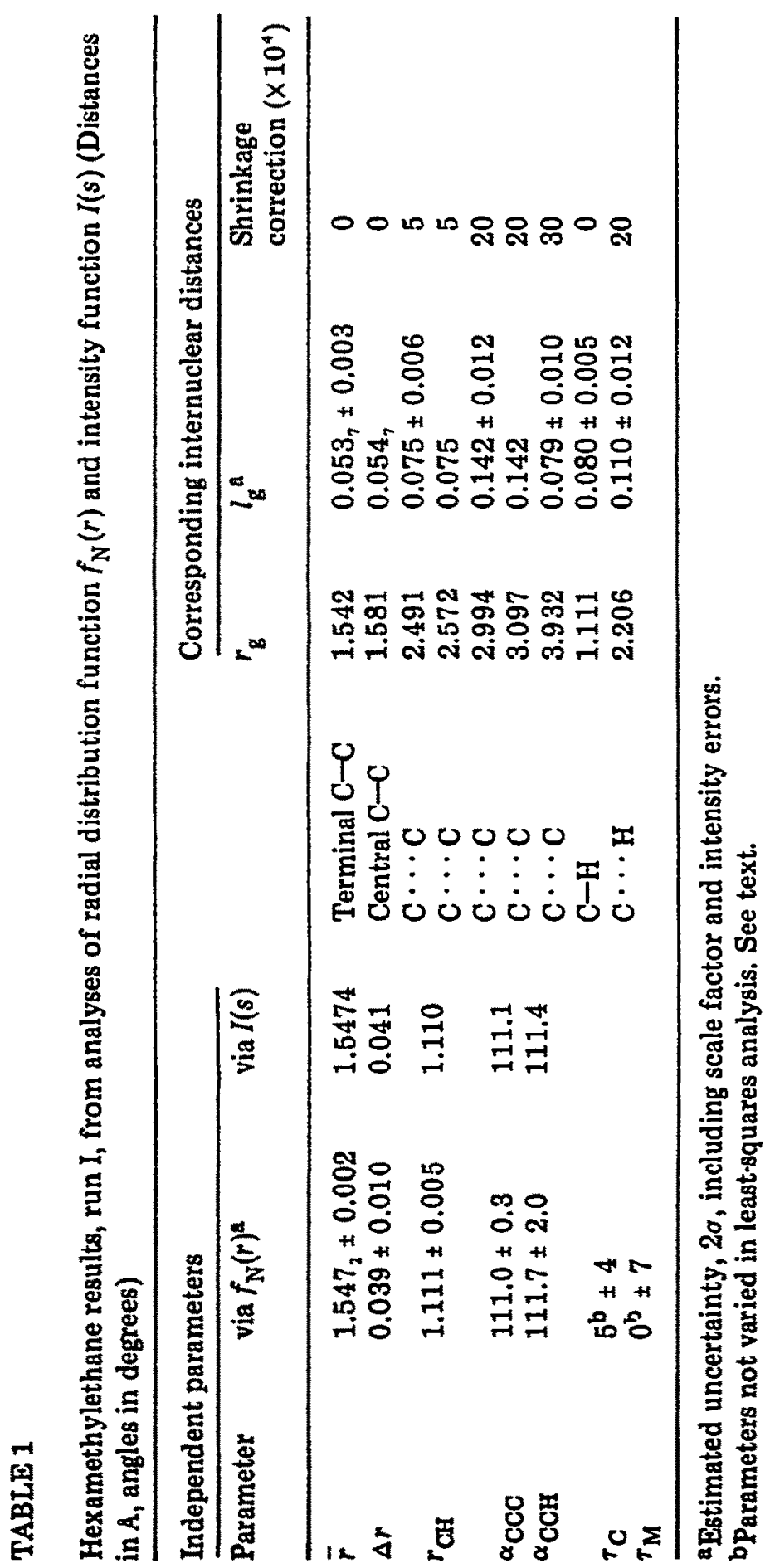









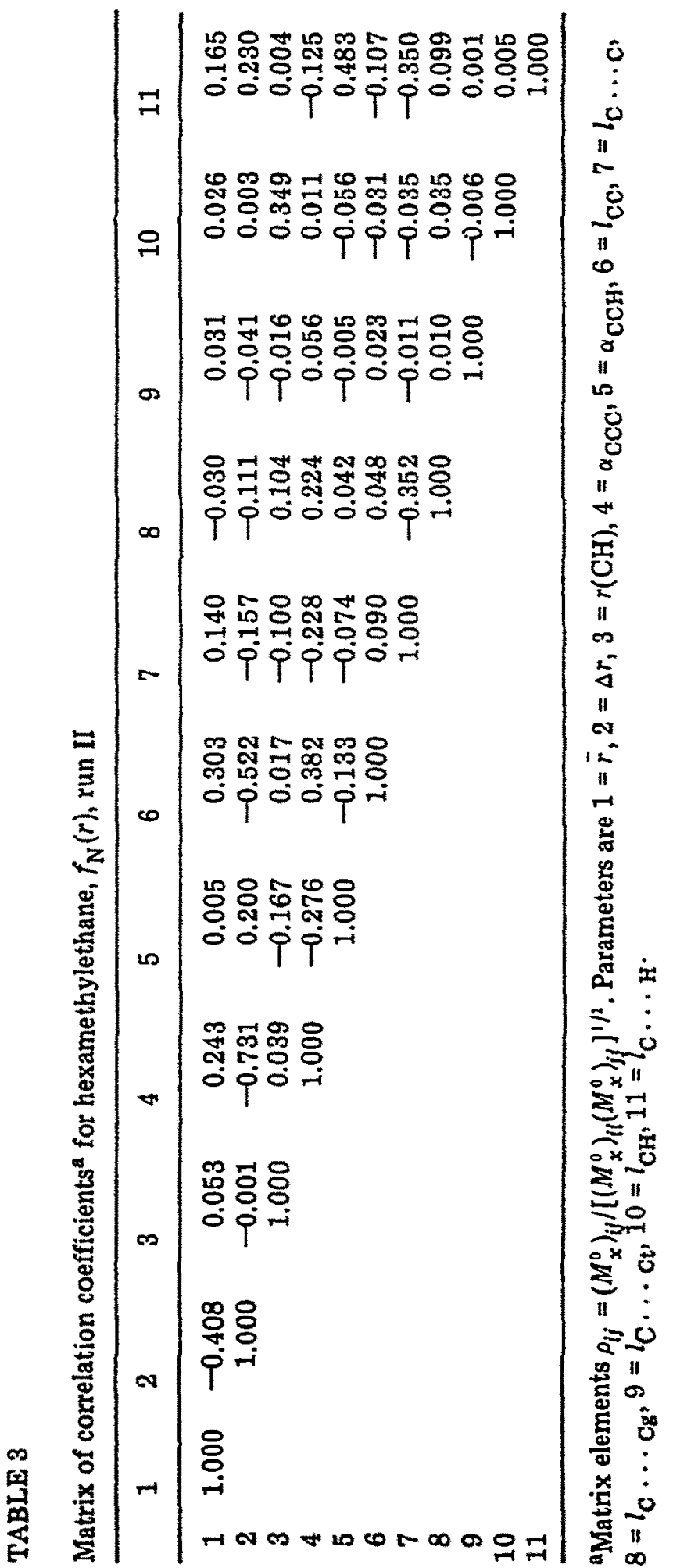




\section{Tetramethylethane}

A gas sample of tetramethylethane at room temperature is a mixture of trans and gauche and, as such, its complete description involves a large number of parameters; therefore, certain assumptions had to be made in order to make the problem tractable. Both isomers were assumed to have the same terminal $\mathrm{C}-\mathrm{C}$ bond lengths, identical $\mathrm{C}-\mathrm{H}$ bond lengths, identical $\mathrm{CCH}$ bond angles and identical $\mathrm{CCC}$ angles between terminal $\mathrm{C}-\mathrm{C}$ bonds. The central $\mathrm{C}-\mathrm{C}$ bond was assumed to be $0.002 \AA$ greater in length in the gauche isomer than in the trans isomer for reasons that will be discussed subsequently. Symmetries of $C_{2 \mathrm{~h}}$ and $C_{2}$ were ascribed to the trans and gauche isomers, respectively. Two different inner angles were considered in the gauche form, namely $C_{1} \vec{C}_{2} C_{3}$ and $C_{1} C_{2} C_{4}$ in which the numbering corresponds to Fig. 4. The $\mathbf{C C H}_{\text {tertiary }}$ bond angles were taken to make the tertiary hydrogens equidistant from each of their three nearest neighbor carbon atoms.

On the basis of the above assumptions, least-squares refinements were performed taking into account the following ten parameters:

(1) the percent of trans and gauche isomers,

(2) $\bar{r}$, the average $\mathrm{C}-\mathrm{C}$ bond length,

(3) $\Delta r$, the difference in length between central $\mathrm{C}-\mathrm{C}$ and terminal $\mathrm{C}-\mathrm{C}$ bonds, in the trans isomer.

(4) $r_{\mathrm{CH}}$, the $\mathrm{C}-\mathrm{H}$ bond length in methyl groups, the tertiary $\mathrm{C}-\mathrm{H}$ bond assumed to be $0.02 \AA$ longer.

(5) $\alpha_{\mathrm{CCC}_{t}}$, the inner CCC bond angle in trans isomer,

(6) $\alpha_{\mathrm{CCC}_{g}}$, the inner $\mathrm{C}_{1} \mathrm{C}_{2} \mathrm{C}_{3}$ bond angle in the gauche isomer,

(7) $\Delta \alpha_{C_{g}}$, the amount by which angle $C_{1} C_{2} C_{3}$ exceeds angle $C_{1} C_{2} C_{4}$,

(8) $\theta_{\mathrm{ccC}}$, the terminal $\mathrm{C}_{3} \mathrm{C}_{2} \mathrm{C}_{4}$ bond angle,

(9) $\alpha_{\mathrm{CCH}}$, the $\mathrm{CCH}$ bond angle and

(10) $\tau_{\mathrm{g}}$, the torsional displacement about the center bond in the gauche isomer, taken as zero in the eclipsed $C_{2 \mathrm{v}}$ form.

It was impossible to obtain convergence when all nine of the structural parameters were allowed to vary simultaneously in least-squares refinements.

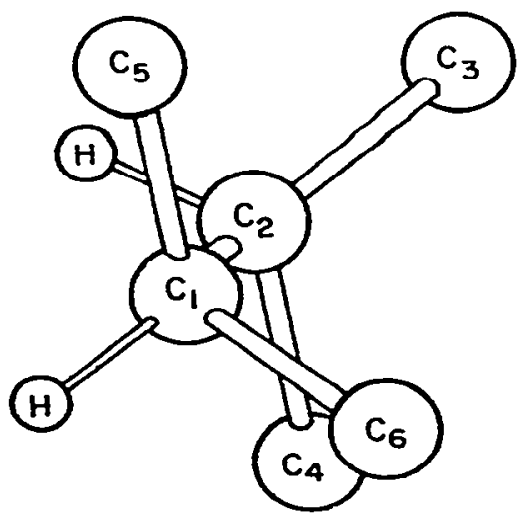

Fig. 4. Atomic numbering in tetramethylethane, gauche conformation. 
The following, imperfect, strategy was applied to obtain a practical solution. The four most subtle parameters, $\Delta r, \theta_{\mathbf{c c c}}, \tau_{\mathrm{g}}$ and $\Delta \alpha_{\mathbf{c c c}}$, were fixed at various values while the other five parameters were refined. Refinements were repeated for a range of fixed values for the four subtle parameters. Plots were made of the standard deviations in the radial distribution functions corresponding to the assumed values of the four preset parameters. Enough points were chosen to characterize the minima in the curves and to show the principal parameter correlations. These plots are shown in Fig. 5. Each curve has identical values for three of the four parameters, $\Delta r, \theta$ ccc, $\tau_{g}$, and $\Delta \alpha_{\mathrm{CCC}_{\mathrm{g}}}$. The parameter specified on the abscissa is the only one varied along a given curve.
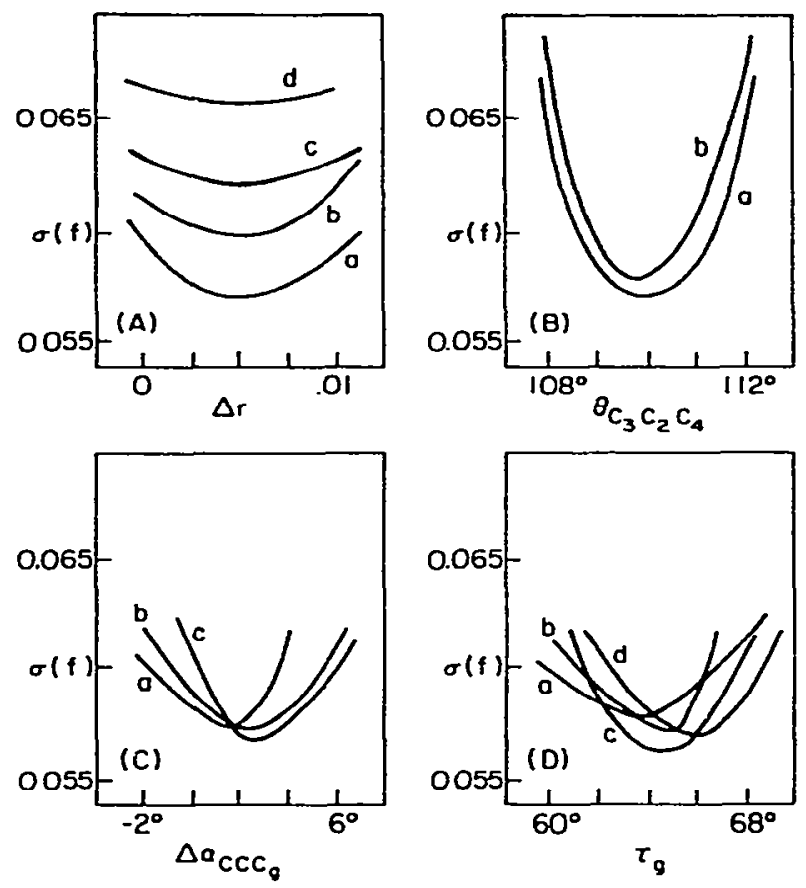

Fig. 5. Closeness of fit of radial distribution function $f_{\mathrm{N}}(r)$ as a function of parameters $\Delta r, \theta, \Delta \alpha$, and ${ }^{\top} g$

(Aa) $\theta=110^{\circ}, \Delta \alpha=2^{\circ}, \tau_{\mathrm{g}}=66.3^{\circ}$.

(Ab) $\theta=110^{\circ}, \Delta \alpha=1^{\circ}, \tau_{g}=66.3^{\circ}$.

(Ac) $\theta=108^{\circ}, \Delta \alpha=1^{\circ}, \tau_{B}=66.3^{\circ}$.

(Ad) $\theta=112^{\circ}, \Delta \alpha=2^{\circ}, \tau_{\mathrm{g}}=66.3^{\circ}$.

(Ba) $\Delta r=0.005 \mathrm{~A}, \Delta \alpha=2^{\circ}, \tau_{\mathrm{g}}=66.3^{\circ}$.

(Bb) $\Delta r=0.005 \mathrm{~A}, \Delta \alpha=0^{\circ}, \tau_{\mathrm{g}}=62.6^{\circ}$.

(Ca) $\Delta r=0.005 \mathrm{~A}, \theta=110^{\circ}, \tau_{\mathrm{g}}=62.6^{\circ}$.

(Cb) $\Delta r=0.005 A, \theta=110^{\circ}, \tau_{g}=64.6^{\circ}$.

(Cc) $\Delta r=0.005 \AA, \theta=110^{\circ}, \tau_{\mathrm{g}}=66.3^{\circ}$.

(Da) $\Delta r=0.005 \AA, \theta=110^{\circ}, \Delta \alpha=0^{\circ}$.

(Db) $\Delta r=0.005 \mathrm{~A}, \theta=110^{\circ}, \Delta \alpha=1^{\circ}$.

(Dc) $\Delta r=0.005 \AA, \theta=110^{\circ}, \Delta \alpha=2^{\circ}$.

(Dd) $\Delta r=0.005 \mathrm{~A}, \theta=110^{\circ}, \Delta \alpha=3^{\circ}$. 
It was found that the weighted average of all the CCC angles was $111.35^{\circ}$, irrespective of the choice of the four fixed parameters. The final models for the two isomers were constructed by constraining the weighted average of all the CCC angles to be $111.35^{\circ}$, and by using values for $\Delta r, \theta_{C c C}, \tau_{g}$, and $\Delta \alpha_{\mathrm{CCC}_{\mathrm{g}}}$ corresponding to the minima in Fig. 5 .

Constraints similar to those used in the hexamethylethane investigation were placed on the amplitudes of vibration in the tetramethylethane analysis. Estimated shrinkage corrections correspond to those listed in Tables 1 and 2.

\section{RESULTS}

The results of the structure determination are presented in Tables 1,2 , and 4 , together with estimated uncertainties $(2 \sigma)$. Intensity correlations were assumed to be characterized by the correlation parameter $\gamma$ with $\gamma \approx 1[10]$. Indices of resolution for hexamethylethane run I were 1.08 for all three camera lengths whereas in run II they were $0.99,1.01$, and 1.00 for the 21-, 11-, and 7-cm camera lengths. Corresponding indices for tetramethylethane were $1.05,1.09$, and 1.05 .

Full details of the refinements of both runs with hexamethylethane can be found in a thesis [11]. Present tabulations differ from those in the thesis in the conversion of standard deviations to a more modern basis [10]. In each run refinements on intensities for each camera distance were consistent with those of the other distances to well within the standard deviations. Refinements of $I(s)$ and $f_{\mathrm{N}}(r)$ gave virtually identical results as can be seen in Tables 1 and 2 . Finally, results of runs $I$ and II agreed with each other somewhat better than expected statistically from the random intensity errors alone and much better than expected from the total propagated errors. Since the runs were made independently with a considerable interval of time between them, there should be little correlation in the estimated scale factor errors. Perhaps error estimates were overly conservative but experience in electron diffraction warns one that hidden systematic errors may perturb results more than can be guessed easily from general considerations. Therefore, it would be prudent to regard the internal consistency in the present work as a pleasing but quite inadequate gauge of the absolute accuracy.

\section{DISCUSSION}

The structures of both molecules revealed features that are readily interpretable as steric deformations. Perhaps the most notable of these occur in the bond lengths of hexamethylethane. A comparison of the $\mathrm{C}-\mathrm{C}$ bonds in hexamethylethane with the $1.537 \AA \mathrm{C}-\mathrm{C}$ bonds found in neopentane [12] indicates a stretching of $0.04_{s} \pm 0.01 \AA$ for the central bond and $0.005 \pm 0.002 \AA$ for the terminal bonds. Presumably these are a consequence of the strong intermethyl repulsions across the central $\mathrm{C}-\mathrm{C}$ bond. The inner angle $\alpha_{\mathrm{ccc}}$ is 
TABLE 4

Tetramethylethane results from analysis of radial distribution function (Distances in $A$, angles in degrees)

\begin{tabular}{|c|c|c|c|c|}
\hline \multicolumn{2}{|c|}{ Independent parameters } & & \multicolumn{2}{|c|}{ Corresponding internuclear distances } \\
\hline Parameter & $r_{g}{ }^{a, b}$ & & $r_{\mathbf{g}}{ }^{2}$ & $l_{\mathrm{g}}{ }^{\mathrm{a}, \mathrm{b}}$ \\
\hline $\bar{r}$ & $1.539 \mathrm{~s} \pm 0.002$ & $\begin{array}{l}(\mathrm{C}-\mathrm{H} \text { methyl) } \\
(\mathrm{C} \cdot \mathrm{H} \text { methyl })^{\mathrm{C}}\end{array}$ & $\begin{array}{l}1.113 \\
2.191\end{array}$ & $\begin{array}{l}0.085 \pm 0.007 \\
0.110 \pm 0.012\end{array}$ \\
\hline$\Delta r$ & $0.005 \pm 0.007$ & trans isomer & & \\
\hline & & Terminal $\mathrm{C}-\mathrm{C}$ & 1.539 & $0.055 \pm 0.003$ \\
\hline & & Central $\mathbf{C}-\mathrm{C}$ & 1.544 & 0.056 \\
\hline$\left(r_{\mathrm{CH}}\right)_{\text {ave }}$ & $1.115 \pm 0.004$ & $\begin{array}{l}\mathbf{C} \cdots \mathbf{C} \\
\mathbf{c} \cdots \mathbf{C}\end{array}$ & $\begin{array}{l}2.522 \\
2.541\end{array}$ & $\begin{array}{l}(0.075 \pm 0.007)^{\mathrm{e}} \\
(0.075)^{\mathrm{e}}\end{array}$ \\
\hline$\alpha_{\mathbf{C C C}_{t}}$ & $111.1 \pm 1.4$ & $\mathbf{C} \cdots \mathbf{C}$ & 2.982 & $0.122 \pm 0.01$ \\
\hline${ }^{\alpha} \operatorname{ccc}_{g}$ & $113.6 \pm 0.9$ & $\begin{array}{l}\text { C } \cdots \text { C } \\
\text { gauche isomerd }\end{array}$ & 3.904 & $(0.075 \pm 0.01)^{e}$ \\
\hline$\Delta \alpha \mathbf{C C C}_{\mathrm{E}}$ & $2 \pm 2$ & $\begin{array}{l}\text { Terminal } \mathrm{C}-\mathrm{C} \\
\text { Central } \mathrm{C}-\mathrm{C}\end{array}$ & $\begin{array}{l}(1.539) \\
(1.546)\end{array}$ & $\begin{array}{l}(0.055)^{f} \\
(0.056)^{f}\end{array}$ \\
\hline${ }^{\theta} \mathrm{C}_{3} \mathrm{C}_{2} \mathrm{C}_{4}$ & $\begin{array}{l}110.1 \pm 0.8 \\
65 \pm 5\end{array}$ & $\begin{array}{l}C_{3} \cdots C_{4} \\
C_{1} \cdots C_{3} \\
C_{1} \cdots C_{4}\end{array}$ & $\begin{array}{l}2.522 \\
2.580 \\
2.550\end{array}$ & $\begin{array}{l}(0.075)^{\mathrm{f}} \\
(0.075)^{\mathrm{f}} \\
(0.075)^{\mathrm{f}}\end{array}$ \\
\hline $\begin{array}{l}\text { Average of } \\
\text { CCC angles }\end{array}$ & $111.3_{s} \pm 0.4$ & $\left.\begin{array}{l}\mathbf{C}_{3} \cdots \mathbf{C}_{5} \\
\mathbf{C}_{3} \cdots \mathbf{C}_{6}\end{array}\right)$ & 3.12 & $\left(0.12_{2}\right)^{f}$ \\
\hline $\begin{array}{l}\text { Average of } \\
\text { inner CCC } \\
\text { angles }\end{array}$ & $112.0 \pm 0.8$ & $\mathrm{C}_{4} \cdots \mathrm{C}_{5}$ & 3.908 & $(0.075)^{f}$ \\
\hline${ }^{\alpha} \mathbf{C C H}$ & $(110.5)^{c}$ & & & \\
\hline
\end{tabular}

"Parameters in parentheses tied to trans parameters in refinements.

${ }^{b}$ Estimated uncertainty, $2 a$.

c Assumes same $r_{\mathrm{CH}}$ as in hexamethylethane, run II.

dResults determined fixing composition at $40 \%$ trans , $60 \%$ gauche, assuming central $\mathrm{C}-\mathrm{C}$ for gauche 0.002 \& longer than for trans.

eTied together.

f Tied to trans values.

opened up by $1.5 \pm 0.3^{\circ}$ in comparison with the tetrahedral angle one would expect around an unstrained quaternary carbon.

The observed deformation of the carbon skeleton from $D_{3 \mathrm{~h}}$ symmetry can also be attributed to a tendency of the methyl groups to avoid each other. In a structure with full $D_{3 h}$ symmetry, six of the methyl hydrogens at one end of the molecule would be projected almost directly at six on the other end that are only about $2 \AA$ away. A slight twist about the central bond significantly increases the $\mathbf{H} \cdots \mathbf{H}$ distances, thereby stabilizing the molecule. Naturally, a twist in one sense is equivalent to one in the other, so that each of the minima in the normal 3-fold barrier potential becomes a double minimum. An alternative interpretation of the diffraction intensities is that the apparent twist from $D_{3 \mathrm{~d}}$ is a torsional shrinkage effect [13]. Unfortunately the diffraction intensities do not discriminate between these two interpretations. 
Semiquantitative calculations via molecular mechanics [2] performed after the structure analysis indicate that the molecule has an equilibrium structure (minimum potential energy) distorted from $D_{3 \mathrm{~d}}$ symmetry by perhaps $13^{\circ}$. A factor complicating a comparison of theory and experiment is the following. Interactions inducing double minima in hexamethylethane would have the effect of flattening the potential well in the region between the minima. This should lead to a greater torsional freedom. It should also distinctly skew the potential in such a way that displacements from the minima are favored if the displacements are toward $D_{3 d}$ symmetry rather than away from it. This should make the mean distortion from $D_{3 \mathrm{~d}}$ less than the equilibrium distortion. Hence, the equilibrium distortion may be greater than the electron diffraction mean distortion of $5^{\circ}$ which looks too small on any basis. In this vein we interject, subjectively, that the central $\mathrm{C}-\mathrm{C}$ bond length looks unrealistically large.

\section{Trimethylamine-trimethylborane complex}

It is worthwhile to speculate on the unresolved structure of $\left(\mathrm{CH}_{3}\right)_{3} \mathrm{BN}\left(\mathrm{CH}_{3}\right)_{3}$ based on the experimental findings for hexamethylethane. Lide [3] interpreted the single rotational constant observed for the amine-borane in terms of a long $1.80 \AA \mathrm{B}-\mathrm{N}$ bond. Geller [4], on the other hand, argued that a normal $1.6 \AA \mathrm{B}-\mathrm{N}$ bond was more likely and that the moment of inertia could be explained by adopting a longer B-C bond of $1.65 \AA$ (as seems reasonable) and assuming an opening of the CBN and CNB angles (perhaps to $113^{\circ}$ or more). Since the force constant for a $B-N$ bond [14] is approximately half that for a $\mathrm{C}-\mathrm{C}$ bond [15], it is tempting to suppose that the steric stretching of the $\mathrm{B}-\mathrm{N}$ bond is roughly twice that of the central bond in hexamethylethane. Similarly, the angles are probably not greatly different from those in hexamethylethane and, therefore, they are probably a little smaller than proposed by Geller. Therefore the structure of $\left(\mathrm{CH}_{3}\right)_{3}$ $\mathrm{BN}\left(\mathrm{CH}_{3}\right)_{3}$ is probably intermediate between the two proposed previously. In all cases but one, Geller's cited B-N bond lengths (which ranged from 1.53 to $1.64 \AA$ ) involved crystalline compounds with $\mathrm{BF}_{3}$ groups. Haaland and co-workers [16] have found with the analogous $\mathrm{X}_{3} \mathrm{AlN}\left(\mathrm{CH}_{3}\right)_{3}$ compounds that gas-phase complexes with $\mathrm{X}=\mathrm{CH}_{3}$ or $\mathrm{H}$ have $\mathrm{Al}-\mathrm{N}$ bond lengths of $0.1 \AA$ greater than do crystalline compounds with $X$ a halogen. In the aluminum compounds with much larger bond lengths steric forces must be small. It is plausible to conclude, tentatively, that the vapor phase complex $\left(\mathrm{CH}_{3}\right)_{3} \mathrm{BN}$ $\left(\mathrm{CH}_{3}\right)_{3}$ does for one reason or another have a $\mathrm{B}-\mathrm{N}$ bond length appreciably greater than those encountered in crystalline $\mathrm{BF}_{3}$ compounds.

\section{Tetramethylethane}

Electron diffraction intensities of tetramethylethane show that approximately $60 \%$ of the vapor phase molecules are in the gauche conformation, a value 
within experimental error of that expected if there is no energetic or vibrational entropic preference for either isomer. This is consistent with spectroscopic investigations which indicate that the energy difference is very small, probably less than $0.1 \mathrm{kcal} \mathrm{mol}^{-1}[17,18]$. It is much smaller, indeed, than the energy difference expected according to the usual rule of thumb making gauche methyl interactions additive. This rule would make the gauche isomer, with 3 such interactions, less stable by about $0.5-0.8 \mathrm{kcal} \mathrm{mol}^{-1}$ [19] than the trans, which possesses only 2 interactions.

Just as $\mathbf{C}-\mathbf{C}$ bonds in hexamethylethane are properly compared with those in neopentane, the bonds in tetramethylethane (mean $r_{\mathrm{g}}(\mathrm{C}-\mathrm{C})=1.540 \pm 0.002 \AA$ $\left(r_{\text {term }}-r_{\text {cent }}\right)=0.005 \pm 0.007 \AA$ for trans $)$ are more naturally compared with those in isobutane $\left(r_{\mathrm{g}}(\mathrm{C}-\mathrm{C})=1.535 \pm 0.002 \AA\right)$ [20]. Evidently the steric stretching displacements in tetramethylethane are much smaller than those in hexamethylethane. Although the former molecule has half (gauche conformer) or one-third (trans conformer) as many destabilizing methyl-methyl interactions as the latter, the steric deformations seem to be even smaller than this ratio suggests. The reason probably lies partly in the $\mathrm{C}-\mathrm{C}-\mathrm{C}$ angles which, in this analog of isobutane $\left(\angle C C C=110.8^{\circ}\right)[20]$, are naturally greater than $109.47^{\circ}$ and, hence, give naturally greater methyl-methyl clearances. Another factor is that methyl torsions in tetramethylethane can relieve intermethyl repulsions whereas, in $D_{3 \mathrm{a}}$ hexamethylethane, they cannot.

A greater stretching of the central bond in the gauche isomer than in the trans isomer may be expected. As mentioned above, the gauche isomer has a larger number (by $50 \%$ ) of intermethyl repulsions acting across the central bond than the trans isomer. This together with preliminary refinements of $\Delta r$, accounts for the constraint imposed in the least-squares refinements whereby the central bond was taken to be $0.002 \AA$ longer in the gauche isomer than in the trans isomer.

The $\mathrm{C}-\mathrm{C}-\mathrm{C}$ bond angles in tetramethylethane are distributed about the isobutane value in a way that is consistent with the steric perturbations. In particular, the fact that methyl groups $C_{6}$ and $C_{3}$ (See Fig. 4) suffer twice as many steric interactions as groups $\mathrm{C}_{4}$ and $\mathrm{C}_{5}$ in the gauche isomer suggests that $\angle C_{1} C_{2} C_{3}$ should be greater than $\angle C_{1} C_{2} C_{4}$. The least-squares refinements are in accord with this argument. The greater opening of the $\mathrm{C}_{1} \mathrm{C}_{2} \mathrm{C}_{3}$ and $\mathrm{C}_{2} \mathrm{C}_{1} \mathrm{C}_{5}$ angles in the gauche isomer could lead to a difference between the various $3.1 \AA$ gauche distances such that the $\mathrm{C}_{3} \cdots \mathrm{C}_{6}$ distance would exceed the $\mathrm{C}_{6} \cdots \mathrm{C}_{4}$ distance. This potential splitting of $3.1 \AA$ gauche distances is offset by an internal rotation of the isomer so that the $\mathbf{C}_{3} \cdots \mathbf{C}_{6}$ and $\mathrm{C}_{6} \cdots \mathrm{C}_{4}$ distances turn out to be approximately equal.

\section{CONCLUSION}

Tetramethylethane and hexamethylethane are good examples of molecules exhibiting steric deformations. Energy minimization calculations (molecular mechanics) by Jacob [2], who invoked a very simple model force field, gave 
a reasonable account of the structural features, bond lengths as well as angles, of both molecules. The day is approaching when semiempirical or even ab initio calculations will be capable of predicting structures with a substantially greater precision than the present experiments. Molecular mechanics calculations certainly offer the cheapest and most practical approach but a satisfactory and reliable model force field still eludes chemists. Its formulation must be based on a wide range of empirical information, including structures of molecules such as tetra- and hexamethylethane.

\section{ACKNOWLEDGEMENTS}

Research supported by grants from the National Science Foundation.

\section{REFERENCES}

1 L. S. Bartell, Trans. Amer. Crystallogr. Assoc., 2 (1966) 134; L. S. Bartell, J. Chem. Educ., 45 (1968) 754.

2 E. J. Jacob, H. B. Thompson and L. S. Bartell, J. Chem. Phys., 47 (1967) 3736.

3 D. R. Lide, R. W. Taft and P. Love, J. Chem. Phys., 31 (1959) 561.

4 S. Geller, J. Chem. Phys., 32 (1960) 1569.

5 L. S. Bartell, in A. Weissberger and B. W. Rossiter (Eds.), Physical Methods in Chemistry, 4th edn., Interscience, New York, 1973.

6 R. M. Gavin and L. S. Bartell, J. Chem. Phys., 48 (1968) 2460.

7 L. S. Bartell and B. L. Carroll, J. Chem. Phys., 42 (1965) 1135.

8 R. M. Badger, J. Chem. Phys., 2 (1934) 128.

9 O. Bastiansen and M. Traetteberg, Acta Crystallogr., 13 (1960) 1108; Y. Morino,

S. J. Cyvin, K. Kuchitsu and T. Iijima, J. Chem. Phys., 36 (1962) 1109.

10 L. S. Bartell and M. G. Anashkin, J. Mol. Struct., 17 (1973) 193.

11 T. L. Boates, Ph.D. Thesis, Iowa State University, 1966.

12 W. F. Bradford, Ph.D. Thesis, University of Michigan, 1975.

13 L. S. Bartell and D. A. Kohl, J. Chem. Phys., 39 (1963) 3097.

14 R. C. Taylor, Advan. Chem. Ser., (1966) 59.

15 J. H. Schachtschneider and R. G. Snyder, Spectrochimica Acta, 19 (1963) 117.

16 G. A. Anderson, F. R. Forgaard and A. Haaland, Acta Chem. Scand., 26 (1972) 1947;

A. Aimenningen, G. Gundersen, T. Haugen and A. Haaland, Acta Chem. Scand., 26 (1972) 3928; O. Gropen and A. Haaland, Acta Chem. Scand., 27 (1973) 521; A. Almenningen, A. Haaland, T. Haugen and D. P. Novak, Acta Chem. Scand., 27 (1973) 1821.

17 P. W. Scott, J. P. McCullough, K. D. Williamson and G. Waddington, J. Amer. Chem. Soc., 73 (1951) 1707.

18 J. K. Brown and N. Sheppard, J. Chem. Phys., 19 (1951) 976.

19 See ref. 2 and the citations therein.

20 R. L. Hilderbrandt and J. D. Wieser, J. Chem. Phys., 56 (1972) 1143. 